\title{
MÉTODO DE FATORAÇÃO LU PARA SOLUÇÃO DE SISTEMAS LINEARES
}

\section{LU FACTORIZATION METHOD FOR SOLVING LINEAR SYSTEMS}

\author{
Natalia Rodrigues da Silva, Fernando Pereira de Souza, Edivaldo \\ Romanini
}

Universidade Federal de Mato Grosso do Sul, Curso de Matemática, cidade Três LagoasMS.

e-mail ntl rodrigues@outlook.com

Agência de fomento: Programa de Educação Tutorial

RESUMO - Na resolução de sistemas lineares é comum analisar não apenas um sistema $A X=B$, mas vários, em que a matriz $A$ é conservada sendo alterado apenas o vetor $B$. O método de Eliminação de Gauss consiste em efetuar transformações elementares sobre as equações de um sistema $A X=B$, até obter um sistema triangular superior $\mathrm{UX}=\mathrm{C}$, equivalente ao sistema dado. Tal método obrigaria a resolver tudo desde o início, para cada novo vetor B(RUGGIERO; LOPES, 1988). Alternativamente, a fatoração LU de $A$, permite que a solução de cada sistema se dê apenas alterando o vetor B. O presente trabalho é resultado de um estudo de iniciação científica do Curso de Licenciatura em Matemática e ligado ao Programa de Educação Tutorial (PET). Neste trabalho, o objetivo é apresentar o método de eliminação de Gauss com pivotamento parcial, o algoritmo para resolução de sistemas lineares triangulares e o método de fatoração LU da matriz dos coeficientes.

Palavras-chave: método de fatoração LU; sistema de equações lineares; matriz triangular inferior; matriz triangular superior; algoritmo.

ABSTRACT - In the resolution of linear systems it is common to analyze not only a system $A X=B$, but several, in which the matrix $A$ is conserved and only the vector $B$ is altered. The Gauss Elimination method consists of performing elementary transformations on the equations of a system $A X=B$ until an upper triangular system $U X=C$, equivalent to the given system. Such a method would oblige us to solve everything from the beginning, for each new vector $B$ (RUGGIERO; LOPES, 1988). Alternatively, the LU factorization of A allows the solution of each system to occur only by changing the vector $B$. The present work is the result of a scientific initiation study of the Degree in Mathematics and linked to the Tutorial Education Program (PET). In this work, the objective is to present the Gauss elimination method with partial pivoting, the algorithm

Recebido em: 09/08/2017 Revisado em: 22/09/2017 Aprovado em: 28/09/2017 for solving triangular linear systems and the LU factorization method of the coefficient matrix.

Keywords: LU factorization method; system of linear equations; unit lower triangular matrix; upper triangular matrix; algorithm. 


\section{INTRODUÇÃO}

A decomposição de uma matriz quadrada $A$ de ordem $n$ na forma $L U$, consiste na fatoração de $\mathrm{A}$ como produto de duas matrizes triangulares, a saber, uma matriz triangular superior $U$ (Upper) e uma matriz triangular inferior L (Lower). A decomposição de uma matriz na forma LU é amplamente empregada na resolução numérica de sistemas de equações lineares quando tratado por métodos diretos. Dentre inúmeras aplicações relacionadas à resolução de sistemas lineares podemos destacar: a obtenção da corrente elétrica num circuito, a resposta numérica de problemas modelados via Equações Diferenciais Parciais e a solução de problemas discretos de sistemas dinâmicos. Na fatoração LU de $A$ a obtenção das matrizes $\mathrm{L}$ e $\mathrm{U}$ pode ser conseguida através do Método de Eliminação de Gauss (MEG). A fim de evitar problemas de estabilidade numérica é aconselhável utilizar o pivotamento parcial durante o MEG. Na decomposição LU de A, a matriz triangular superior $U$ conterá em suas entradas os valores decorrentes do processo de eliminação de Gauss. Já a matriz triangular inferior L será constituída de uma diagonal principal unitária $\left(\mathrm{l}_{\mathrm{ii}}=1, \quad \forall \quad\right.$ i) e dos multiplicadores $m_{\mathrm{ij}}$ situados abaixo dessa diagonal unitária. Os multiplicadores também são obtidos durante 0 processo de eliminação de Gauss. Vale destacar que a utilização do pivotamento parcial, que consiste em colocar na diagonal principal o elemento de maior magnitude durante o processo de escalonamento, faz com que os multiplicadores fiquem restritos ao intervalo $[-1,1]$, garantindo, dessa forma, estabilidade numérica quando for realizada a resolução do sistema triangular inferior. Na resolução numérica de um sistema linear $A X=B$, em que o vetor B sofre variações, sendo mantida inalterada a matriz dos coeficientes, torna-se muito vantajosa à estratégia da fatoração LU ao invés da resolução numérica direta via decomposição de Gauss; pois no primeiro caso (fatoração LU) a decomposição será realizada uma única vez.

\section{METODOLOGIA}

No desenvolvimento deste trabalho foram realizados estudos teóricos sobre a resolução de sistemas lineares e fatoração LU. Foram analisados algoritmos convencionais ligados à resolução de sistemas lineares triangulares. Tais algoritmos foram aplicados para resolução de um problema de maximização (CHINCHOLE; BHADANE, 2014). Para uma melhor compreensão do assunto abordado, foram feitas apresentações de seminários com a presença do orientador. 


\section{RESULTADOS}

\section{Método de Eliminação de Gauss}

Este método consiste em transformar um sistema linear num sistema linear equivalente, sendo a matriz final triangular. Sistemas lineares em que a matriz dos coeficientes é triangular apresentam algoritmos de fácil resolução. Para modificar um sistema linear dado de forma a obter um sistema equivalente, faremos uso do seguinte teorema:

Teorema 1: Seja $A X=B$ um sistema linear. Aplicando sobre as equações deste sistema uma sequência de operações, escolhidas entre:
a) Trocar duas equações.
b) Multiplicar uma equação por uma constante não nula.
c) Adicionar um múltiplo de uma equação a outra equação.

Obtemos um novo sistema $\tilde{A} X=\tilde{B}$ e os sistemas $A X=B \quad$ e $\quad \tilde{A} X=\tilde{B} \quad$ são equivalentes. A fim de ilustrar o Teorema 1, consideremos o seguinte exemplo (RUGGIERO; LOPES, 1988):

Exemplo 1: Seja o sistema linear

$$
\left\{\begin{array}{c}
3 x_{1}+2 x_{2}+4 x_{3}=1 \\
x_{1}+x_{2}+2 x_{3}=2 \\
4 x_{1}+3 x_{2}-2 x_{3}=3
\end{array}\right.
$$

A matriz associada ao sistema linear (1)é dada por

$$
A^{(0)} \mid B^{(0)}=\left(\begin{array}{ccc|c}
3 & 2 & 4 & 1 \\
1 & 1 & 2 & 2 \\
4 & 3 & -2 & 3
\end{array}\right)
$$

Estágio 1: Verificar a necessidade do pivotamento. Notemos que o maior elemento da primeira coluna é $a_{31}=4$, desta forma faremos,

$$
L_{1} \leftrightarrow L_{3} \Rightarrow A^{(0)} \mid B^{(0)}=\left(\begin{array}{ccc|c}
4 & 3 & -2 & 3 \\
1 & 1 & 2 & 2 \\
3 & 2 & 4 & 1
\end{array}\right)
$$

Agora, devemos eliminar $x_{1}$ das equações 2 e 3 :

$$
\underset{\substack{L_{2} \leftrightarrow L_{2}-1 / L_{1} \cdot L_{1} \\
L_{3} \leftrightarrow L_{3}-3 / 4 \cdot L_{1}}}{L_{1}} A^{(1)} \mid B^{1}=\left(\begin{array}{ccc|c}
4 & 3 & -2 & 3 \\
0 & 1 / 4 & 5 / 2 & 5 / 4 \\
0 & -1 / 4 & 22 / 4 & 5 / 4
\end{array}\right)
$$

Os multiplicadores do estágio 1 são $m_{21}=1 / 4$ e $m_{31}=3 / 4$.

Estágio 2: Verificar a necessidade do pivotamento. Se não for o caso, eliminação de $x_{2}$ na equação 3 . Dessa forma,

$$
\begin{gathered}
L_{1} \leftrightarrow L_{1} \\
L_{2} \leftrightarrow L_{2} \\
L_{3} \leftrightarrow L_{3}+L_{2}
\end{gathered} \quad \Rightarrow A^{(2)} \mid B^{2}=\left(\begin{array}{ccc|c}
4 & 3 & -2 & 3 \\
0 & 1 / 4 & 5 / 2 & 5 / 4 \\
0 & 0 & 32 / 4 & 0
\end{array}\right)
$$

assim, o multiplicador do estágio 2 é $m_{32}=1$. Resolver o sistema linear (1) é o mesmo que resolver o sistema equivalente,

$$
\left\{\begin{array}{c}
4 x_{1}+3 x_{2}-2 x_{3}=3 \\
1 / 4 x_{2}+5 / 2 x_{3}=5 / 4 \\
32 / 4 x_{3}=0
\end{array}\right.
$$

Portanto, $x_{3}=0$, que nos dá $x_{2}=5$ e $x_{1}=-3 ;$ logo temos o vetor solução $X=(-3,5,0)^{T}$. Vejamos um algoritmo para resolução de sistemas lineares triangulares.

\section{Resolução de Sistemas Lineares Triangulares}

Consideremos o sistema linear triangular superior, em que $a_{i i} \neq 0$, para $\mathrm{i}=1,2, \ldots, \mathrm{n}$. 


$$
\left\{\begin{array}{c}
a_{11} x_{1}+a_{12} x_{2}+a_{13} x_{3}+\cdots+a_{1 n} x_{n}=b_{1} \\
a_{22} x_{2}+a_{23} x_{3}+\cdots+a_{2 n} x_{n}=b_{2} \\
a_{33} x_{3}+\cdots+a_{3 n} x_{n}=b_{3} \\
\ddots \\
a_{n n} x_{n}=b_{n}
\end{array}\right.
$$

Da última equação, temos $x_{n}=$ $b_{n} / a_{n n}$. A variável $x_{n-1}$ pode ser obtida da penúltima

equação: $x_{n-1}=\left(b_{n-1}-a_{n-1, n} x_{n}\right) / a_{n-1, n-1}, \mathrm{e}$

assim

sucessivamente

obtemos $x_{n-2}, x_{n-3, \cdots}, x_{2} \quad$ e finalmente $x_{1}: x_{1}=\left(b_{1}-a_{12} x_{2}-\cdots-a_{1, n} x_{n}\right) / a_{11}$.

Portanto, obtemos o seguinte algoritmo (RUGGIERO; LOPES, 1988):

$$
\begin{aligned}
& x_{n}=\frac{b_{n}}{a_{n n}}, \\
& \operatorname{para} k=n-1, \cdots, 3,2,1 \\
& x_{k}=\left(b_{k}-\sum_{j=k+1}^{n} a_{k j} \cdot x_{j}\right) / a_{k k}
\end{aligned}
$$

A seguir, vamos analisar um método para resolver sistemas lineares em que a matriz dos coeficientes não é necessariamente triangular.

\section{Fatoração $\boldsymbol{L U}$}

Como dito anteriormente, a fatoração $L U$ da matriz dos coeficientes apresenta vantagens quando comparada com a resolução numérica via eliminação de Gauss com pivotamento parcial. Além disso, sistemas lineares triangulares são fáceis de resolver.A seguir vamos detalhar o esquema da fatoração $L U$ de $A$. Suponhamos que seja possível escrever,

$$
A=L U
$$

em que $L$ é uma matriz triangular inferior e $U$ é uma matriz triangular superior. Uma vez encontrada as matrizes $L$ e $U$, o sistema linear $A X=B$ pode ser reescrito como,

$$
A X=B \Rightarrow(L U) X=B \Rightarrow L(U X)=B \text {. }
$$

Se $Y=U X$, então, resolver o sistema linear $A X=B$, é equivalente a resolver o sistema linear $L Y=B$, seguido da resolução do sistema linear $U X=Y$.Como comentado anteriormente, os fatores $L \mathrm{e} U$ podem ser obtidos utilizando o Método de Eliminação de Gauss com pivotamento parcial.

A fim de ilustrar a metodologia estudada consideremos o seguinte problema de programação linear, a saber,

Maximizar a função $z=c_{1} x_{1}+c_{2} x_{2}+$ $\cdots+c_{n-1} x_{n-1}$, sujeito a restrições

$$
\left\{\begin{array}{c}
a_{21} x_{1}+a_{22} x_{2}+\cdots+a_{2, n-1} x_{n-1} \leq b_{2} \\
a_{31} x_{1}+a_{32} x_{2}+\cdots+a_{3, n-1} x_{n-1} \leq b_{3} \\
\vdots \\
a_{n 1} x_{1}+a_{n 2} x_{2}+\cdots+a_{n, n-1} x_{n-1} \leq b_{n}
\end{array}\right.
$$

em que $x_{1}, x_{2}, \cdots, x_{n-1} \geq 0$. O objetivo é encontrar a variável $z$, que satisfaz o sistema $A X=B$ onde,

$$
\begin{gathered}
A=\left(\begin{array}{cccc}
a_{11} & \cdots & a_{1, n-1} & 1 \\
a_{21} & \cdots & a_{2, n-1} & 0 \\
\vdots & \ddots & \vdots & \vdots \\
a_{n 1} & \cdots & a_{n, n-1} & 0
\end{array}\right), \quad X=\left(\begin{array}{c}
x_{1} \\
x_{2} \\
\vdots \\
x_{n-1} \\
z
\end{array}\right), \\
B=\left(\begin{array}{c}
0 \\
b_{2} \\
\vdots \\
b_{n-1} \\
b_{n}
\end{array}\right),
\end{gathered}
$$

Em que $a_{11}=-c_{1}, \cdots, a_{1, n-1}=$ $-c_{n-1}$. Consideremos um exemplo de ordem 4, a fim de ilustrar o método de fatoração $L U$. 
Exemplo 2: Maximizar a função $z=3 x_{1}+5 x_{2}+4 x_{3}$ sujeita as restrições (CHINCHOLE; BHADANE, 2014),

Seja então,

$$
2 x_{1}+3 x_{2} \leq 8,2 x_{2}+5 x_{3} \leq 10,3 x_{1}+2 x_{2}+4 x_{3} \leq 15, \quad x_{1}, x_{2}, x_{3} \geq 0 \text {. }
$$

$$
A=\left(\begin{array}{cccc}
-3 & -5 & -4 & 1 \\
2 & 3 & 0 & 0 \\
0 & 2 & 5 & 0 \\
3 & 2 & 4 & 0
\end{array}\right)
$$

Os multiplicadores do estágio 1 do processo são,

$$
m_{21}=a_{22}^{(0)} / a_{11}^{(0)}=-2 / 3, m_{31}=a_{31}^{(0)} / a_{11}^{(0)}=0, \quad m_{41}=a_{41}^{(0)} / a_{11}^{(0)}=-1 .
$$

Para eliminar $x_{1}$ da linha $j, j=2,3$, multiplicamos a linha 1 por $m_{j 1}$ e subtraímos o resultado da linha $j$. Assim,

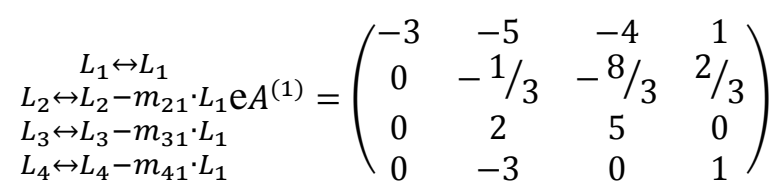

Os multiplicadores do estágio 2 são dados por,

$$
m_{32}=a_{32}^{(1)} / a_{22}^{(1)}=-6, \quad m_{42}=a_{42}^{(1)} / a_{22}^{(1)}=9 .
$$

Teremos,

$$
\underset{\substack{L_{1} \leftrightarrow L_{1} \\
L_{2} \leftrightarrow L_{2} \\
L_{3} \leftrightarrow L_{3}-m_{32} \cdot L_{2} \\
L_{4}-L_{4}-m_{42} \cdot L_{2}}}{\text { e }} A^{(2)}=\left(\begin{array}{cccc}
-3 & -5 & -4 & 1 \\
0 & -1 / 3 & -8 / 3 & 2 / 3 \\
0 & 0 & -11 & 4 \\
0 & 0 & 24 & -5
\end{array}\right)
$$

Os multiplicadores do estágio 3 são dados por, $m_{43}=a_{43}^{(3)} / a_{33}^{(3)}=-24 / 11$. Desta forma temos,

$$
\begin{aligned}
& L_{1} \leftrightarrow L_{1} \\
& L_{2} \leftrightarrow L_{2} \\
& L_{3} \leftrightarrow L_{3} \\
& L_{4} \leftrightarrow L_{4}-m_{43} \cdot L_{3}
\end{aligned} \quad \text { e } A^{(3)}=\left(\begin{array}{cccc}
-3 & -5 & -4 & 1 \\
0 & -1 / 3 & -8 / 3 & 2 / 3 \\
0 & 0 & -11 & 4 \\
0 & 0 & 0 & 41 / 11
\end{array}\right)
$$

os fatores $L$ e $U$ são, 


$$
L=\left(\begin{array}{cccc}
1 & 0 & 0 & 0 \\
-2 / 3 & 1 & 0 & 0 \\
0 & -6 & 1 & 0 \\
-1 & 9 & -24 / 11 & 1
\end{array}\right), \quad U=\left(\begin{array}{cccc}
-3 & -5 & -4 & 1 \\
0 & -1 / 3 & -8 / 3 & 2 / 3 \\
0 & 0 & -11 & 4 \\
0 & 0 & 0 & 41 / 11
\end{array}\right)
$$

Agora consideremos $L Y=B$, onde

assim, aplicando o algoritmo,

$$
Y=\left(\begin{array}{l}
y_{1} \\
y_{2} \\
y_{3} \\
y_{4}
\end{array}\right), \quad B=\left(\begin{array}{c}
0 \\
8 \\
10 \\
15
\end{array}\right),
$$

$$
\begin{aligned}
& y_{1}=\frac{b_{1}}{l_{11}}, \\
& \quad \begin{array}{c}
\text { para } k=2,3,4 \\
y_{k}=\left(b_{k}-\sum_{j=1}^{k-1} a_{k j} \cdot y_{j}\right) / l_{k k}
\end{array}
\end{aligned}
$$

encontramos

$$
y_{1}=0, y_{2}=8, y_{3}=58, y_{4}=765 / 11 \text {. }
$$

Finalmente a solução,

$$
X=\left(\begin{array}{l}
x_{1} \\
x_{2} \\
x_{3} \\
x_{4}
\end{array}\right)
$$

será obtida da equação $U X=Y$. Desta forma, o algoritmo:

$$
\begin{aligned}
& x_{4}=\frac{y_{4}}{u_{44}}, \quad \text { para } k=3,2,1 \\
& x_{k}=\left(y_{k}-\sum_{j=k+1}^{4} u_{k j} \cdot x_{j}\right) / u_{k k},
\end{aligned}
$$

fornece a solução,

$$
x_{1}=89 / 41, x_{2}=50 / 41, x_{3}=62 / 41, z=x_{4}=765 / 41 \text {. }
$$

Observemos que no exemplo 2, a fatoração $L U$ de $A$ foi realizada sem a estratégia do pivotamento parcial. Quando o mesmo é realizado, torna-se necessário prémultiplicar, por matrizes de permutação, as matrizes que estão sendo transformadas pelo processo gaussiano.

\section{DISCUSSÃO}

O presente estudo mostra a importância da análise de alguns prérequisitos oriundos da Álgebra Linear. 
Através deles que é possível uma melhor compreensão sobre os métodos diretos na resolução de sistemas lineares. Neste contexto, nosso principal interesse neste trabalho foi compreender a resolução de sistemas lineares pela fatoração LU de uma matriz. Esta é uma pesquisa em andamento, sendo este trabalho a etapa inicial de algo mais abrangente, que é desenvolver um algoritmo para a resolução de um sistema linear $\mathrm{AX}=\mathrm{B}$.

\section{CONCLUSÃO}

O estudo do método de fatoração LU, permitiu desenvolver um trabalho com vários conceitos matemáticos ainda não vistos no curso de Licenciatura em Matemática. Este trabalho nos permitiu observar a vantagem do método de fatoração LU sobre o método de eliminação de Gauss.

\section{REFERÊNCIAS}

CHINCHOLE, S. M.; BHADANE, A. P. LU Factorization method to solve linear programming problem. International Journal of Emerging Technology and Advanced Engineering, v. 4, n. 4, abril 2014.

RUGGIERO, M. A. G.; LOPES, V. L. R. Cálculo numérico aspectos teóricos e computacionais, Rio de Janeiro: McGraw Hill, 1988. 